Celestinesca 3I (2007): 119-132 https://doi.org/10.7203/Celestinesca.31.20073

\title{
El error retórico de la alcahueta. Performatividad y nueva retórica en la Celestina
}

\author{
Albert Lloret \\ The Johns Hopkins University
}

A las obras creo, que las palabras de balde las venden dondequiera

(Celestina, Auto viI)

\section{Antiguos modelos y nueva retórica}

Aunque cada vez parezca haber menos duda de que la Celestina sea una obra escrita desde las convenciones de la comedia humanística coetánea al texto (María Rosa Lida, Francisco Rico), no por eso deja de presentar unas características técnicas que se irán desarrollando en obras posteriores, como el Lazarillo o el Quijote, y que, desde el presente, se pueden ver en tendencia hacia la novela moderna de los siglos XVIII y XIX (Stephen Gilman, Dorothy Severin). ${ }^{1}$ De cualquier modo, en la comedia humanística y en la "novela sentimental», con la que tanto tiene que ver el texto que nos ocupa (Severin, Marina Brownlee), las obras solían recibir el título de la pareja de amantes protagonistas, o de uno de ellos. A pesar de que la compleja historia textual de la obra haya podido favorecer una denominación homónima al personaje no de ninguno de los amantes, sino de la alcahueta, si se sigue prefiriendo llamar la Celestina a la Tragicomedia de

1.- Cifrada esencialmente, en su hibridismo genérico y en su dialogismo estilístico integrador de distintos discursos (cómico, irónico, paródico). Ver además el ponderado resumen de Ruiz Arzálluz en Lobera et al. (xcii-cvii). 
Calisto y Melibea — como se debiera y reivindica Joseph Snow ("Sobre la caracterización» y «Two Melibeas») - , es fundamentalmente por la talla del personaje y por su función en el desarrollo de la obra. En palabras de Carlos Mota: «si Celestina adquiere una estatura colosal frente al resto del elenco [de personajes] es porque posee una avasalladora voluntad de dominio: sobre sí misma, sobre todos los demás personajes, sobre la naturaleza» (Lobera et al. cxcii).

Esta voluntad de dominio tiene su puesta en escena más espectacular y artificiosa en la invocación del demonio y en la philocaptio de las que Celestina se sirve a fin de ganar a Melibea para Calisto, al final del tercer auto. ${ }^{2}$ No obstante, en la obra, el proceder de la alcahueta hechicera no resulta de encadenar conjuros con encantamientos. Como sintetiza Malcom Read: "her manipulation of the supernatural power is more than matched by her purely rhetorical skill. Her greatest art is that of controlling others not with witchcraft but with her own linguistic ability" (85). De hecho, para Dorothy Severin, el desproporcionado tamaño retórico que adquiere el personaje podría suscitar una interpretación censora: "Celestina's behaviour is a powerful condemnation of the ability of language, literature and received wisdom to be used as a tool to corrupt and debase» (67, ver también Paul Friedman 360-1).

El poder retórico de Celestina no es una cualidad inherente y exclusiva del personaje, más bien era ya considerado contemporáneamente como parte del arsenal de instrumentos propio del oficio de alcahueta. Como recuerda Malcom Read, Suárez de Figueroa, en su Plaza universal recogía el dicho que: "Menos entiende de persuasión un Retórico que un alcahuete» (84-5). No obstante, la retórica de un «alcahuete» no parece tener relación directa con la disciplina de raíces clásicas, aquella ars bene dicendi que asiste en una buena expresión, correlato del valor moral del rétor, para el convencimiento del oyente en materias de interés público (Lausberg 83-5).

Cuando se ha analizado la Celestina desde el punto de vista de su retórica, se ha tendido a entenderla como una técnica de escritura, un código de tópicos y figuras en boga durante el tiempo en que vivió el autor de la obra (Charles Fraker). ${ }^{3}$ Este código sin duda ha dejado una serie de huellas en el texto, que hoy, por la caída en desuso y desconocimiento de dicho arte, nos son difícilmente reconocibles, lo que nos impide entender la Celestina como lo harían sus contemporáneos; es de suponer: de aquellos que compartían formación y conocimientos con sus creadores. Con esta concepción del arte retórico, Fraker ensaya un catálogo de tópicos y

2.- Ver ahora los trabajos de Robert Folger, Images in Mind y «Passion and Persuasion».

3.- Singularmente en el libro de Fraker, pero también en el trabajo de Erica Morgan y menos en el de Otis Handy. 
figuras (diallage, comparatio, dissimilitudo...) a partir del primer auto de la Celestina, pero lo desestima al poco:

It would be impossible in this brief study to list by genera and species all the figures, topics of invention, or forms of argument in Celestina. More than that, it is unnecessary: any student wealthy enough to own a Quintilian or a Lausberg can point out figures as well. Rhetorical argument in Celestina is in any case pervasive: the proportion of the text that disputes, argues, convinces according to the old patterns is very large $(73$, la cursiva es mía).

Suscribiendo y partiendo del alegato reivindicativo de Fraker respecto la retoricidad del texto y la importancia que la retórica clásica tuvo en la formación de los autores de la Celestina, la aproximación reconstructiva de los recursos empleados, como ensayó y desestimó el mismo Fraker, parece poco productiva. Un estudio retórico del texto tal vez deba centrarse en una determinada escena, en uno o varios de sus personajes, en un auto, en definitiva, una fracción de la obra. Puesto que las artes retóricas construyen discursos, y en una obra dialógica, como lo es ésta, debe haber forzosamente varios, parecería especialmente productivo examinar la construcción de uno de ellos, o el diálogo conflictivo entre más de uno.

En este sentido son modélicos los estudios de Morgan y Handy, que se han ocupado, principalmente, del estudio de los éxitos retóricos de la alcahueta en la seducción de Melibea. Ambos críticos ponen al descubierto las numerosas tretas de las que Celestina se sirve para conducir a Melibea según sus intenciones: argumentos patéticos, entimemas, falsas analogías, o la trasposición de códigos amorosos en otros de tipo médico, socialmente aceptables. ${ }^{4}$

El siguiente trabajo no va a servirse directamente de los «antiguos modelos", sino que abrirá el marco de estudio de la argumentación y la persuasión, sirviéndose, en primer lugar, de los estudios neoretóricos de Chaim Perelman, los cuales asumen y actualizan el saber clásico. En efecto, según la aproximación de Perelman, recurrir sólo a los «antiguos modelos» puede resultar un inconveniente en algunas circunstancias: La retórica, convertida en ejercicio escolar, se dirige a auditorios convencionales y puede, sin dificultad alguna, atenerse a visiones estereotipadas

4.- Robert Folger ha descifrado recientemente el sentido fisiológico de la estratagema de Celestina según las teorías al uso contemporáneo («Passion and Persuasion», especialmente 16-24); mientras que Friedman ha reevaluado el análisis retórico de la persuasión de Melibea por parte de la alcahueta desde el post-estructuralismo, señalando la cesión de toda lógica y de toda retórica consecuentes, y la sumisión de un lenguaje aceptable socialmente a la satisfacción de las propias intenciones y deseos. Por otro lado, Di Patre ha puesto de relieve los usos lógicamente contradictorios de las sentencias. 
de estos auditorios, lo cual ha contribuido, tanto como lo facticio de sus temas, a su degeneración. (55)

En otras palabras: parte de las estratagemas retóricas de Celestina serán difíciles de apreciar con propiedad si se olvida que la alcahueta no se dirige en ningún momento al senado o a un jurado, sino a personajes individuales, específicos, caracterizados ficticiamente, por cuestiones alejadísimas de aquellas por las que la retórica clásica desarrolla sus ejercicios.

Lo que despliega la "nueva retórica», rescatando el saber clásico de los limitados contextos de aplicación originales, es el conocimiento de todas las condiciones y medios argumentativos para conseguir el objetivo de toda ars bene dicendi, la persuasión, esté o no basada en las preceptivas antiguas, como es el caso de aquélla que Sánchez de Figueroa recuerda como habilidad de los alcahuetes, en absoluto formados escolarmente en este saber. En palabras de Perelman:

El objetivo de toda argumentación (...) es provocar o acrecentar la adhesión a las tesis presentadas para su asentimiento: una argumentación eficaz es la que consigue aumentar esta intensidad de adhesión de manera que desencadene en los oyentes la acción prevista (acción prevista o abstención) o, al menos, que cree en ellos una predisposición, que se manifestará en el momento oportuno. (91)

El enfoque global y los objetivos últimos de Perelman sobrepasan en mucho mis intenciones. Perelman, en la Europa posterior a las dos grandes guerras, reivindicaba el fundamento ético de la construcción de un discurso práctico sobre el saber humano, más allá del prejuicio de raíz cartesiana que había venido concediendo valor ontológico solamente a aquello que ya era en sí mismo evidente. Revalorizando el uso argumentativo de la palabra, Perelman abogaba por una herramienta agonística refinada por siglos de civilización, alternativa deseable a la barbarie de las armas, terreno abierto al encuentro de posturas no absolutas sino necesariamente matizadas, a la búsqueda del diálogo, en favor de la persuasión y el convencimiento último. En este proceso, Perelman define e ilustra un elenco de topoi y figuras de argumentación no codificadas por las venerables artes de la antigüedad, que pueden arrojar nueva luz sobre aquellos textos cuya dimensión persuasoria es notable.

Así pues, lo que pretendo a continuación es entender un poco mejor el arte retórico de un personaje ficticio caracterizado por su capacidad de persuadir a sus interlocutores. Para ello pretendo analizar la que - como escribía María Rosa Lida - es la "piedra de toque» del arte persuasivo celestinesco: su dominio sobre Pármeno. Seguidamente me centraré en la derrota y la muerte de Celestina a manos de Pármeno y Sempronio, 
para averiguar cuáles fueron los errores retóricos de la alcahueta, cuyas palabras no pudieron impedir el trágico desenlace.

\section{Palabras y performatividad}

El proceso celestinesco de dominio sobre Pármeno empieza hacia el final del primer auto. ${ }^{5}$ Hasta ese momento, Pármeno le había mostrado a su señor Calisto, sin éxito alguno, una desfavorable opinión de Celestina, a quien conocía y había servido tiempo atrás. También Sempronio y Celestina, cuando se encuentran presentes, se dan por apercibidos de la desfavorable opinión que Pármeno manifiesta sobre ellos en sus apartes. Celestina, para cambiar la postura de Pámerno, tal y como reza el argumento del auto, «mucho le dice de los hechos y conocimiento de su madre [de Pármeno], induciéndole a amor y concordia de Sempronio» (I, 25). En esta frase se encuentra tanto la sustancia del diálogo entre ambos personajes en el primer auto, como el objetivo de la argumentación de Celestina y el argumento en el que ésta se basa principalmente. Se trata de convencer a Pármeno de que ayude a Sempronio - $y$, por lo tanto, a sí misma y a sus intereses-, invocando la figura de la madre de Pármeno, aprovechando que ésta y Celestina habían sido comadres en el oficio ${ }^{6}$

Se puede empezar a observar cuáles son las condiciones argumentativas que se dan en esta situación: se trata de un diálogo, en el que uno de sus dos participantes pone en juego sus habilidades retóricas con el objetivo último de persuadir al otro. El éxito del ejercicio dialéctico se cifrará en una acción muy concreta, implicará, siguiendo la terminología de Perelman, una "acción consecuente», en este caso, la colaboración de Pármeno con Sempronio. El auditorio de la argumentación no es universal sino particular, y concretísimo, compuesto de una sola persona (Pármeno), con una predisposición poco favorable al asentimiento de las palabras salidas por la boca de la alcahueta, en función del conocimiento del pasado y la fama de Celestina (Perelman 65-71).

Siendo así, y guiándonos por lo que el argumento del auto declara, se materializa una operación importantísima no únicamente en un proceso judicial (Perelman 192), sino en cualquier defensa de unos determinados intereses, y su consecuente argumentación: la selección de los hechos que

5.- Ver las páginas definitivas de Gilman, Lida y José Antonio Maravall sobre la evolución de Pármeno, vital, psicológica y en función de la relación jerárquica con su amo, respectivamente. Una lectura integradora que orquestra las perspectivas anteriores a la vez que pone cierta atención en el papel celestinesco dentro del proceso en Snow, "¿Con qué pagaré esto?».

6.- En el tercer auto puede leerse una interpretación de lo acontecido en el diálogo de éste primero, en el que intervienen Celestina y Pármeno. La alcahueta cree que sus intenciones persuasorias se vieron materializadas como podemos adivinar cuando dice: «acordele [a Pármeno] quién era su madre» (III, 99-100). 
apoyan las intenciones de Celestina. Así, el hecho de que Celestina recuerde a Pármeno quién había sido su madre, parece obrado en clara conciencia del auditorio unipersonal al que persuadir, buscando hábilmente, pues, la sensibilidad del receptor a los argumentos escogidos.

¿Por qué es un argumento a favor de las intenciones celestinescas que la madre de Pármeno hubiera sido la compañera de Celestina? La elección de esta información es clave y tiene muchas aristas. En primer lugar, le permite a la alcahueta recortar distancias sociales y morales con Pármeno ( $i$ Pues fuego malo te queme, que tan puta vieja era tu madre como yo!»; I, 71), e intentar interponer espacio entre Pármeno y Calisto («deja los vanos prometimientos de los señores, los cuales desechan la sustancia de los sirvientes con huecos y vanos prometimientos»; I, 73). De esta manera, en virtud de este vínculo familiar, justifica la necesidad de que Pármeno ayude a Sempronio en su propio beneficio, sin cuidado del perjuicio que pueda aportar a su amo. ${ }^{7}$ En el fondo, pues, la base argumentativa de la alcahueta en este punto es la figura de la doble jerarquía (Perelman 340-43): del mismo modo que tu madre recibía tu lealtad, Pármeno, sin menoscabo de sus actividades profesionales que condenas en mí, debes serme leal y seguir mis consejos, ya que yo también soy para ti como una madre.

Paralelamente, Celestina juega la carta de la autoridad y del saber que otorga la experiencia, opuesta a la juventud del criado ( $i \mathrm{O}$ h hijo!, bien dicen que la prudencia no puede ser sino en los viejos, y tú mucho mozo eres»; I, 74), estrategia que converge con la de detentar la familiaridad de Pármeno:

los ancianos somos llamados padres, y los buenos padres bien aconsejan a sus hijos, y en especial yo a ti, cuya vida y honra más que la mía deseo. Y ¿cuándo me pagarás tú esto? Nunca, pues a los padres y a los maestros no puede ser hecho servicio igualmente. (I, 78-9)

El efecto buscado con esta estrategia cae en uno de los topoi comunes del orden (Perelman 160), por lo menos implícitamente, reafirmando la superioridad de lo anterior - Celestina, su experiencia y saber - sobre lo posterior -Pármeno, su inocencia y su prudencia impropias de la juventud-. Por otro lado, este topos del orden da cuenta de los usos de Séneca o de las Auctoritates Aristotelis en las palabras de Celestina que se acaban de citar en el párrafo anterior, donde se traducen las sentencias "Magistri, diis et parentibus non potest reddi equivalens", o "Iuvenes non possunt prudentes esse» en refuerzo de la autoridad de la vieja ante los reparos de Pármeno (ver también Di Patre, 158-64).

7.- Puntualmente, por lo tanto, Celestina se contradice, puesto que había empezado por decir a Pármeno que ella sólo estaba ayudando a su amo para la consecución de algo natural (I, 67-9). 
Es ésta una estrategia de algún modo opuesta a la de enfatizar su familiaridad pretérita con Pármeno. Le permite a Celestina extender sus peroratas contra la prudencia o sobre la necesidad de compartir los placeres, pero la oposición argumentativa que adopta en estas circunstancias interpone distancia entre las partes en vez de acortarla:

Mientras que el creador debe, normalmente, adaptarse al auditorio, no sucede lo mismo con el maestro encargado de enseñar a sus alumnos lo que está permitido en el grupo concreto al que deben agregarse o, al menos, al que las personas responsables de su educación desean integrarlos. La persuasión es, en este caso, previa a la iniciación, y debe someterse a las exigencias del grupo especializado cuyo maestro aparece como portavoz. (Perelman 169).

Teniendo en cuenta, entonces, que Pármeno no reconocía desde un principio la autoridad de Celestina, sino todo lo contrario — de ahí la necesidad de la persuasión-, parecen a priori poco convenientes los argumentos de la alcahueta que explotan esta posición. ${ }^{8}$

Finalmente, Celestina recurre a dos argumentos de un tipo peculiar, no codificado por Perelman. A este tipo lo llamaré argumento performativo, y se caracteriza por materializar lingüísticamente, la propia acción que enuncia (Austin 1-24). Su efecto va algo más allá del puro acto lingüístico y obliga al enunciante a establecer un compromiso de acción futura. Los dos argumentos performativos que usa Celestina son la promesa del dinero que su padre le había legado para que se traspasara cuando fuera conveniente, y la del cuerpo de Areúsa.

Así pues, las maniobras que emprende Celestina en este primer auto son, por un lado, el de la figura de la doble jerarquía, el topos de precedencia (en contradicción relativa con los efectos conseguidos con la maniobra anterior), las figuras de autoridad y, finalmente, dos argumentos performativos, dos promesas. ${ }^{9}$ Sin embargo, no porque podamos entender cuáles son los resortes de las intenciones celestinescas significa que sean efectivos. Debemos examinar cuáles son las reacciones de Pármeno, para determinar la efectividad de cada treta. Para decirlo con Perelman:

8.- José Ángel Alonso, en un técnico y riguroso análisis semiótico de la manipulación de Celestina, sigue a Greimas y Courtés para explicar el cambio de lealtad de Pármeno por las estructuras modales y contractuales que se arman en las relaciones entre Pármeno y Calisto, y entre Pármeno y Celestina. Sin embargo, la formalización de dicho estudio no se ocupa de detallar, como se pretende conseguir en éste, los mecanismos que permiten las relaciones entre los personajes, es decir, las relaciones retóricas manifiestas en el texto.

9.- Ver Azar para una interesante valoración general del uso de las promesas en la obra desde la speech act theory, pero sin relacionarlas con las estratagemas argumentativas. Se pone especial atención en los actos performativos, pero no en los actos lingüísticos constativos ("acordele quién era su madre», "bien dicen que la prudencia no puede ser sino en los viejos», etc.), que también intervienen en las maniobras que pretenden provocar asentimiento en los personajes-interlocutores de la obra. 
la situación argumentativa, esencial para la determinación de los lugares a los que se recurrirá, es propiamente un complejo que comprende, a la vez, el objetivo perseguido y los argumentos con los que se corre el riesgo de enfrentarse. (165)

El primer argumento al que parece asentir relativamente Pármeno es el que deriva de su familiaridad con Celestina y de su distancia social y económica con el amo Calisto, pues justo cuando acaba de exponérselo la vieja, el jovenzuelo responde:

Celestina, todo tremo en oírte; no sé qué haga; perplejo estó. Por una parte téngote por madre, por otra a Calisto por amo. Riqueza deseo, pero quien torpemente sube a lo alto, más aína cae que subió. No querría bienes mal ganados. (I, 74)

Pármeno expresa su deseo de medrar, pero también sus prevenciones morales, que refuerza aún más cuando Celestina replica que ella sí que querría bienes mal ganados, porque es de la opinión que: "A tuerto o a derecho, nuestra casa hasta el techo» (I, 74).

La otra reacción que hace mella en el ánimo de Pármeno es la promesa de Areúsa ("Maravillosa cosa es»; I, 76), respecto cuya veracidad Pármeno responde con recelo ( $\mathrm{Mi}$ fe, madre, no creo a nadie»; I, 76); si bien esta vez la autoridad aristotélica ("Estremo es creer a todos y yerro no creer a ninguno»; I, 76) parece ser efectiva, puesto que Pármeno responde: «Digo que te creo pero no me atrevo, déjame» (I, 76). Pármeno sigue presentando resistencia, pero, finalmente, tras el discurso sobre la necesidad de compartir el goce - otro argumento basado en la autoridad profesoral del orador-, Celestina parece haberse salido con la suya: «manda, que a tu mandado mi consentimiento se humilla» (I, 80).

En conclusión, hasta aquí, si bien parecía que los argumentos celestinescos podían resultar lógicamente contradictorios, resultan relativamente efectivos en el auditorio unipersonal al que van dirigidos. En particular, la combinación de autoridad aristotélica y promesa lujuriosa empieza a desarmar al interlocutor. Celestina, a pesar de las palabras de Pármeno, aún no lo considera leal («yo le contaré en el número de los míos»; III, 101) y lo cierto es que, en el auto sexto, el sirviente vuelve a levantar suspicacias en las palabras de la alcahueta frente a Calisto. Ante la situación ya prevista por la vieja, el siguiente movimiento también lo anuncia con anticipación: «Harele haber a Areúsa», (III, 102). ${ }^{10}$

10.- Emilio Barón analiza detalladamente la transformación de Pármeno como una estricta mímesis psicológica, como proceso de maduración personal y afloración de la auténtica naturaleza psicológica (rencorosa, hipócrita, codiciosa, cobarde y lujuriosa), teniendo en cuenta el papel de Celestina, como agente que suscita la autoconsciencia del personaje y su 


\section{Más allá de la performatividad}

Por lo tanto, a pesar de todas las tretas y de la argumentación de la vieja, efectiva en apariencia, no es ya la promesa sino su consecución, lo único que el personaje juzga factible para lograr la lealtad de Pármeno. No es la palabra, tampoco la palabra performativa, sino la acción misma, lo que en el mundo gobernado por un maestro de la persuasión puede desencadenar otra acción. Escribía a este respecto Read:

Not surprisingly, all the characters are deeply suspicious of language, especially those like Celestina who are largely instrumental in its perversion. She is never in doubt as to language's pure symbolic role. (94)

Esta última afirmación la ilustra Read con dos frases de la vieja alcahueta: "dile que cierre la boca y comience a abrir la bolsa» (I, 66) y "que la amistad que entre ti e mí se afirma no ha menester preámbulos ni correlarios ni aparejos para ganar voluntad» (I, 51). Otras podrían aducirse, entre ellas, la que encabeza este artículo (VII, 177): «A las obras creo, que las palabras de balde las venden dondequiera». Cualquiera de ellas demuestra que Celestina conoce muy bien los límites efectivos del lenguaje. ${ }^{11}$

Después del fracaso de la primera conversación con Pármeno, patente en el auto sexto, Celestina habla con él de nuevo en el auto séptimo, abundando en los mismos puntos abordados en su primera conversación: su relación familiar («hijo a lo menos cuasi adotivo»; vII, 163), su autoridad («mira que soy vieja y el buen consejo mora en los viejos»; vII, 163) y, lo que resulta ser determinante, la promesa de nuevo de Areúsa. Para fomentar la concordia con Sempronio, en consecuencia, la vieja promete a Pármeno no ser menos con él: “¡Oh cuán dichosa me hallaría en que tú y Sempronio estuviésedes muy conformes, muy amigos, hermanos en todo, viéndoos venir a mi pobre casa a holgar, a verme, y aun a desenojaros con sendas mochachas!» (VII, 167).

En este punto, surge Calisto una vez más en la conversación, de boca de Pármeno e inopinadamente ( «le consejaba yo lo cierto y me daba malas gracias»; VII, 167), lo que, por un lado, es indicio de que Pármeno está aceptando la autoridad más que la familiaridad de Celestina, a cambio de la promesa de Areúsa y, por otro lado, certifica que Pármeno es consciente de que secundar a Celestina es ir contra Calisto, por mucho que haya

maduración, pero sin atender a las formas ni las causas de la efectividad de las estrategias celestinescas.

11.- El perceptivo análisis de Mary Gaylord, en particular 5-8, parece reforzar nuestras apreciaciones, al centrarse en demostrar el cariz comercial o económico del lenguaje, utilizado por los personajes en la obra como medio para la obtención de todo tipo de beneficios. Por nuestra parte, compartimos las apreciaciones de Gaylord aunque tendemos a precisarlas y naturalizarlas dentro de la casuística de un fenómeno intrínsecamente comunicativo, como es el de la persuasión, en el sentido amplio que venimos empleando. 
dicho la vieja poco antes: «estando bien con él, con tu amo y con todo el mundo lo estarías» (VII, 165).

Es aquí cuando Pármeno entra en la lógica argumental de Celestina, empezando por aceptar la familiaridad y el vínculo de servidumbre que con ella mantuvo en el pasado: "Agora doy por bien empleado el tiempo que siendo niño te serví, pues tanto fruto trae para la mayor edad» (VII, 168). Pero esta actitud es adoptada sólo jocosamente por parte del sirviente, ya que ante el elogio de Claudina, Pármeno exclama en un aparte: "No la medre Dios más a esta vieja, que ella me da placer con estos loores de sus palabras» (VII, 169). ${ }^{12}$ Aun más: al ver Pármeno a Areúsa, cuando la promesa pasa a ser un hecho no lingüístico que revierte en otro - la lealtad a Sempronio y temporalmente a ella-, el sirviente no puede reprimir en un tono claramente paródico del de la poesía de cancionero o de la fi ción sentimental: «que me ha muerto de amores su vista. Ofrécele cuanto mi padre te dejó para mí» (VII, 181). Reaparece aquí el dinero, del que no se había vuelto a hablar, una de las tretas más groseras y poco sutiles de la alcahueta. Pármeno parece poner en juego con ironía su valor simbólico, precisamente ante aquella persona que se lo había otorgado, la única para la que podía tenerlo.

Por lo tanto, parece claro que Pármeno adopta el discurso de Celestina, se amolda a él, a sus argumentos, para proponer una justificación discursiva que le permita llevar a cabo sus propios fines, de acuerdo con las promesas de la vieja. Él, como Celestina, se sirve de las palabras confiá doles un valor simbólico y pervertible. La supuesta pericia dialéctica de la alcahueta solamente resulta contra Pármeno en tanto que cimentada en argumentos performativos, en promesas, que implican una consecución de determinadas acciones para poder conseguir otras, facilitar su encuentro sexual con Areúsa, a cambio de la concordia con Sempronio.

\section{La muerte de la alcahueta}

Y es aquí donde reside el fracaso celestinesco. Cuando Sempronio y Pármeno aparecen de madrugada, en el doceno auto, ahora son ambos los que se introducen de lleno en el juego retórico al que quería jugar aquélla: "Abre, que son tus hijos» (la cursiva es mía, 254). Las intenciones de los dos sirvientes son claras. Celestina ha conseguido beneficios del negocio en el que participó gracias a Sempronio, y éstos, ahora amigos y hermanos, quieren parte de los beneficios, como fue acordado. La réplica de Celestina ante las demandas no oculta sus intenciones: "iGracioso es el asno!» (XII, 256). A partir de aquí, las múltiples razones contradicto-

12.- Una caracterización de Pármeno como pícaro, y su degeneración rufianesca en John Wilhite. 
rias argüidas de nada pueden servir: se desdice de su obligaciones como nueva ama de los dos siervos, dice haber perdido la cadenilla, o que se la robaron, afirma que lo que le fue dado es suyo y de nadie más (XII, 256), promete, de nuevo contradictoriamente, calzas a cada uno si la cadenilla aparece, pero dice que dicha promesa es de «buen amor» (XII, 257), cuando justo antes se había zafado de las exigencias de los siervos aduciendo que las había hecho de "buen amor» y que, por ello, no estaba obligada a nada. Les promete también otras mujeres (XII, 258) y, a partir de aquí, al ver la ira de los siervos, juega la carta del pathos e intenta suscitar la compasión presentándose como una pobre viejecilla indefensa (XII, 259), pero no admite estar a la merced de Pármeno a raíz de sus conocimientos sobre su pasado, ya que Pármeno repite que él había sabido siempre cómo era la vieja.

Podría parecer que si Celestina pierde esta vez es porque no sabe argumentar como es debido su postura, y su postura es la de mantener todo el capital que ha conseguido con el negocio, en parte gracias al pacto con los criados. Celestina se deja dominar por la codicia y no cede ni una de las cien monedas de oro que le diera Calisto. Podríamos aceptar, con Perelman que:

el hombre apasionado, cuando argumenta, lo hace sin prestar la atención suficiente al auditorio al que se dirige: llevado por el entusiasmo (...). Lo que la pasión provoca es menos una ausencia de razones que una mala elección de las razones. (61)

La reflexión de Perelman es idónea para entender el momento que acabamos de describir siempre que aceptemos una concepción más amplia de lo que significa un argumento. Un argumento que lleva a la persuasión y a la consecución de otra acción puede ser asimismo una promesa u otra acción misma no-lingüística. En la situación de Celestina con los dos sirvientes, el único argumento válido hubiera sido el cumplimiento de parte de las promesas de enriquecimiento mutuo. Cumplir promesas: como había hecho con Pármeno y de la misma manera que había podido obtener beneficios de Calisto presentándole pruebas de sus acciones. Apasionada por la codicia, la vieja no argumentó con aquello que hubiera podido salvarle la vida, descuidando un principio del universo moral de la Tragicomedia que Pármeno recuerda en todo momento: el hecho de que las palabras "de balde las venden dondequiera». ${ }^{13}$

13.- Este artículo está en deuda con la clases de retórica de Pere Ballart en Bellaterra, y con los seminarios sobre la Celestina de Joachim Küpper y Marina Brownlee en Baltimore. Agradezco la generosa y minuciosa lectura de Joseph Snow, y las atentas observaciones de dos lectores de Celestinesca; todas ellas han mejorado sustancialmente este trabajo. Naturalmente, el autor es el único responsable de los aciertos y de todos los errores que pueda haber en el texto. 


\section{Obras citadas}

Alonso Aldama, José Ángel. "A Problem of Narrative Interaction in La Celestina». Signs of Humanity. Proceedings of the Fourth International Congress, International Association for Semiotic Studies. Berlín: Mouton de Gruyter, 1992. 687-91.

Austin, John L. How to Do Things With Words. Cambridge, Mass.: Harvard UP, 1975.

AzAR, Inés. "Speech Act Theory on Self, Responsibility and Discourse». Homenaje a Ana María Barrenechea. Eds. Lía Schwartz Lerner e Isaías Lerner. Madrid: Castalia, 1984. 33-40.

Baron Palma, Emilio. «Pármeno: la liberación del ser auténtico. El antihéroe». Cuadernos Hispanoamericanos 317 (1976): 383-400.

Brownlee, Marina. The Severed Word. Ovid's Heroides and the "Novela Sentimental». Princeton: Princeton UP, 1990.

Di Patre, Patrizia. «P y NO-P. El lenguaje retórico de La Celestina». Celestinesca 29 (2005): 155-169.

Folger, Robert. «Passion and Persuasion: Philocaption in La Celestina». La Corónica 34.1 (2005): 5-29.

-. Images in Mind. Lovesickness, Spanish Sentimental Fictiona don Don Quijote. Chapel Hill: U of North Carolina P, 2002.

Fraker, Charles. Celestina: Genre and Rhetoric. Londres: Tamesis, 1990.

FRIEDMAn, Edward. «Rhetoric at Work. Celestina, Melibea and the Persuasive Arts». Fernando de Rojas and Celestina: Approaching the Fifth Centenary. Eds. Ivy A. Corfis and Joseph T. Snow. Madison: Hispanic Seminary of Medieval Studies, 1993. 359-70.

GaYlord, Mary Malcom. «Fair of the World, Fair of the Word: The Commerce of Language in La Celestina». Revista de Estudios Hispánicos 25.1 (1991): 1-27.

Gilman, Stephen. The Art of La Celestina. Madison: The U of Wisconsin P, 1956.

HANDY, Otis. "The Rhetorical and Psychological Defloration of Melibea». Celestinesca 7.1 (1983): 17-27.

Lausberg, Heinrich. Manual de retórica literariria. Fundamentos de una ciencia de la literatura. Trad. José Pérez Riesco. 3 vols. Madrid: Gredos, 1975.

Lida de Malkiel, María Rosa. La originalidad artística de La Celestina. Buenos Aires, EUDEBA, 1962.

Lobera, Francisco J., Serés, Guillermo, Díaz-Mas, Paloma, et al. «Prólogo». Rojas (2000). li-ccl.

Maravall, José Antonio. El mundo social de La Celestina. Madrid: Gredos, 1972.

Morgan, Erica. "Rhetorical Technique in the Persuasion of Melibea». Celestinesca 3.2 (1979): 7-18. 
Perelman, Chaim y Olbrechts-Tyteca, L. Tratado de la argumentación. Trad. Julia Sevilla Muñoz. Madrid: Gredos, 1989.

ReAD, Malcolm. "The Rhetoric of Social Encounter: La Celestina and the Renaissance Philosophy of Language». The Birth and Death of Language: Spanish Literature and Linguistics (1300-1700). Madrid: Porrúa-Turanzas, 1983. 70-96.

Rico, Francisco. «La realidad y el estilo (El humanismo de La Celestina)». Rojas (2000) xv-xlvii.

RojAS, Fernando de, y Antiguo autor. La Celestina. Tragicomedia de Calisto y Melibea. Eds. Francisco J. Lobera, Guillermo Serés, Paloma Díaz-Mas, Carlos Mota, Íñigo Ruiz Arzálluz y Francisco Rico. Barcelona: Crítica, 2000.

SeVERIN, Dorothy Sherman. Tragicomedy and Novelistic Discourse in Celestina. Cambridge: Cambridge UP, 1989.

Snow, Joseph T. «Sobre la caracterización de Calixto y Melibea». Imago Hispaniae: Homenaje a Manuel Criado de Val. Kassel: Reichenberger, 1989. 185-92.

-. "¿Con qué pagaré esto? The Life and Death of Pármeno"». The Age of the Catholic Monarchs (1474-1516). Literary Studies in Memory of Keith Winnhom. Eds. Alan Deyermond y Ian Macpherson. Liverpool: Liverpool UP, 1989. 185-192.

-. "Two Melibeas». Nunca fue pena mayor. Estudios de Literatura Española en homenaje a Brian Dutton. Eds. A Menéndez Collera y V. Roncero López. Cuenca: U de Castilla-La Mancha, 1996. 655-62.

Stamm, James R. «El tesoro de Pármeno». La Celestina y su contorno social. Actas del I Congreso Internacional sobre La Celestina. Barcelona: Borrás, 1977. 185-91.

Wilhite, John F. «Fernando de Roja's Pármeno: The Making of a Pícaro». South Atlantic Bulletin 41.2 (1976): 137-44. 


\section{LLORET, Albert, «El error retórico de la alcahueta. Performatividad y nueva retórica en la Celestina», Celestinesca 31 (2007), pp. 119-132.}

\section{RESUMEN}

Este trabajo examina las artes retóricas de Celestina en dos episodios de la obra: la persuasión de Pármeno, en palabras de María Rosa Lida, la piedra de toque de las artes celestinescas; y los momentos previos a su muerte, es decir, cuando se produce el fracaso de sus capacidades de dominio sobre las voluntades ajenas. Desde una perspectiva retórica que parte de los modelos clásicos pero los adapta a maniobras argumentativas no codificadas por los tratadistas de la antigüedad, es decir, a la luz del tratado de argumentación de Chaim Perelman, se ponen de relieve puntos clave de las estratagemas celestinescas y el principal error retórico que la conduce a la muerte.

PALABRAS CLAVE: Celestina, retórica, performatividad, Chaim Perelman

\section{ABSTRACT}

This article examines Celestina's rhetorical arts in two episodes of the work: the persuasion of Pármeno - in María Rosa Lida's words, the touchstone of Celestinesque persuasive arts-, and the moments prior to her death - namely, the time when her skills in dominating others' wills fail. From a rhetorical stand-point that stems from Classical models, but readapts them to explain argumentative maneuvers not codified in ancient treatises (thus, in light of Chaim Perelman's treatise on argumentation), several stratagems of the old bawd are underscored, and also the main rhetorical mistake that leads to her death.

KEY WORDS: Celestina, rhetoric, performativity, Chaim Preleman

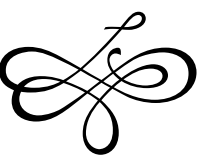

